\title{
Is improvement in the Healthy Food Intake Index (HFII) related to a lower risk for gestational diabetes?
}

\author{
Jelena Meinilä ${ }^{1 *}$, Anita Valkama ${ }^{1,2}$, Saila B. Koivusalo ${ }^{3}$, Beata Stach-Lempinen ${ }^{4}$, Kristiina Rönö ${ }^{3}$, \\ Jaana Lindström ${ }^{5}$, Hannu Kautiainen ${ }^{1,6}$, Johan G. Eriksson ${ }^{1,2,5}$ and Maijaliisa Erkkola ${ }^{7}$ \\ ${ }^{1}$ Department of General Practice and Primary Health Care, University of Helsinki and Helsinki University Hospital, \\ Helsinki 00014, Finland \\ ${ }^{2}$ Folkhälsan Research Center, Helsinki 00250, Finland \\ ${ }^{3}$ Department of Obstetrics and Gynecology, University of Helsinki and Helsinki University Hospital, Helsinki OoO29, Finland \\ ${ }^{4}$ Department of Obstetrics and Gynecology, South-Karelia Central Hospital, Lappeenranta 53130, Finland \\ ${ }^{5}$ Department of Chronic Disease Prevention, National Institute for Health and Welfare, Helsinki 00271, Finland \\ ${ }^{6}$ Department of General Practice and Primary Health Care, University of Eastern Finland, Kuopio 70211, Finland \\ ${ }^{7}$ Department of Food and Environmental Sciences, University of Helsinki, Helsinki O0014, Finland
}

(Submitted 8 September 2016 - Final revision received 7 March 2017 - Accepted 6 April 2017 - First published online 24 May 2017)

\section{Abstract}

The aim was to analyse whether changes in the Healthy Food Intake Index (HFII) during pregnancy are related to gestational diabetes (GDM) risk. The 251 pregnant women participating had a pre-pregnancy BMI $\geq 30 \mathrm{~kg} / \mathrm{m}^{2}$ and/or a history of GDM. A $75 \mathrm{~g}$ oral glucose tolerance test (OGTT) was performed during the first and second trimesters of pregnancy for assessment of GDM. A normal OGTT result at first trimester was an inclusion criterion for the study. FFQ collected at first and second trimesters served for calculating the HFII. A higher HFII score reflects higher adherence to the Nordic Nutrition Recommendations (NNR) (score range 0-17). Statistical methods included Student's $t$ test, Mann-Whitney $U$ test, Fisher's exact test and linear and logistic regression analyses. The mean HFI at first trimester was 10.1 (95\% CI 9.7, 10.4) points, and the mean change from the first to the second trimester was 0.35 (95\% CI $0.09,0.62)$ points. The range of the HFII changes varied from -7 to 7 . The odds for GDM decreased with higher HFII change (adjusted OR 0.83 per one unit increase in HFII; $95 \%$ CI $0.69,0.99 ; P=0.043$ ). In the analysis of the association between HFII-sub-indices and GDM, odds for GDM decreased with higher HFII-Fat change (fat percentage of milk and cheese, type of spread and cooking fats) but it was not significant in a fully adjusted model $(P=0.058)$. Dietary changes towards the NNR during pregnancy seem to be related to a lower risk for GDM.

Key words: Dietary assessment: Lifestyle changes: Dietary changes: Nutrition and diabetes

The prevalence of gestational diabetes (GDM) is increasing globally and is currently affecting $4-15 \%$ of pregnant women in Western countries ${ }^{(1,2)}$. Modifiable risk factors may include diet and physical activity, which have also been the primary targets of most intervention studies ${ }^{(3)}$. Even though most lifestyle intervention studies have failed in preventing GDM, we recently showed that a lifestyle intervention with diet and physical activity counselling can prevent GDM in high-risk women ${ }^{(4)}$. At about the same time, Jing et al. ${ }^{(5)}$ also succeeded in preventing GDM with diet and physical activity counselling. According to epidemiological cohort studies, dietary factors seem to be strongly associated with GDM risk ${ }^{(6,7)}$. Observational studies have reported that a Western dietary pattern associates with an elevated risk for GDM, and dietary patterns characterised by high consumption of vegetables, fruit, dietary fibre and low consumption of high-fat/high-sugar foods and red and processed meat associate with a lower risk for $\mathrm{GDM}^{(7-10)}$. However, a prospective study focusing on the relationship between an actual dietary change during pregnancy and GDM risk is still lacking.

Measuring changes in overall diet instead of performing traditional individual-nutrient/food analysis is justified because, first, several dietary factors seem to be associated with GDM risk $^{(11)}$. Second, a change in one dietary factor is usually followed by a compensatory change in another factor, and these changes cannot and should not be separated ${ }^{(12)}$. Third, subtle changes in the intake of individual nutrients or foods may become detectable only when several changes are combined $^{(13)}$. Diet-quality indices can be suitable for measuring changes in overall diet ${ }^{(14)}$. Several dietary patterns measured by

Abbreviations: GDM, gestational diabetes; HFII, Healthy Food Intake Index; LTPA, leisure time physical activity; NNR, Nordic Nutrition Recommendations; OGTT, oral glucose tolerance test.

* Corresponding author: J. Meinilä, email jelena.meinila@helsinki.fi 
indices, such as Mediterranean dietary pattern, alternate Mediterranean dietary pattern, Diet Approach to Stop Hypertension and alternate Healthy eating index, associate with $\operatorname{GDM}^{(7,8)}$. However, studying the association between a change in diet-quality index score and GDM risk is a novel approach.

Culturally and locally acceptable dietary patterns for GDM prevention are essential. Specific Nordic cultural characteristics of diets are taken into account in food-based guidelines of the Nordic Nutrition Recommendations $(\mathrm{NNR})^{(15)}$. A diet adherent to the NNR has characteristics similar to those of the aforementioned healthy diets associated with GDM. The similarities with the other dietary indices are high intake of fruit, berries, vegetables, whole-grain foods, nuts and legumes, and low intake of $\mathrm{Na}$, red and processed meats and sugar-sweetened beverages. The NNR also recommends vegetable oils, such as rapeseed oil and vegetable margarines, instead of animal fats, as well as low-fat instead of high-fat dairy products ${ }^{(15)}$. A recently developed and validated diet-quality index, the Healthy Food Intake Index (HFII), measures adherence of a diet to the $\mathrm{NNR}^{(16)}$. The aim of the current study was to analyse whether changes in the HFII, and concurrently changes in the adherence to the NNR, from the first to the second trimester of pregnancy is related to GDM among women at a high risk for GDM. A further aim was to study whether any sub-index within the HFII drives/influences the possible relationship between the HFII and GDM.

\section{Methods}

The study participants were part of the Finnish Gestational Diabetes Prevention Study (RADIEL), a multicentre lifestyle intervention study conducted in two Southern Finnish districts, namely the Helsinki Metropolitan area and Lappeenranta, between 2008 and 2014. A total of 496 (68\% of all the women assessed for eligibility) Finnish women with an elevated risk for GDM due to obesity (BMI $\geq 30 \mathrm{~kg} / \mathrm{m}^{2}$ ), a history of GDM, or both participated. They were either $<20$ weeks pregnant ( $n$ 293) or were planning pregnancy ( $n$ 203). These women were allocated to a control ( $n$ 236) or an intervention ( $n$ 260) group. Exclusion criteria were age $\leq 18$ years, diabetes diagnosed before pregnancy, use of medication influencing glucose metabolism, multiple pregnancy, physical disability, current substance abuse, severe psychiatric disorder and substantial communication difficulties. The participants in the control group received standard antenatal care whereas those in the intervention group participated in diet and physical activity counselling. Depending on the recruitment timing (pre- or during pregnancy) the counselling started before pregnancy or at early pregnancy. The diet intervention was given by a dietitian, and it emphasised a high intake of fruit, vegetables, high-fibre grains and fish and a low intake of high-fat dairy products and meat and high-energy/low-nutrient foods, as well as choosing vegetable fat over animal fat ${ }^{(17)}$. Physical activity counselling encouraged moderate-intensity physical activities for a minimum of $150 \mathrm{~min} /$ week and an overall active lifestyle. In the present study, forty-five (18\%) participants were recruited before pregnancy and 206 (82\%) participants in early pregnancy. The control ( $n$ 119) and the intervention ( $n$ 132) groups were combined to study whether an actual change in dietary pattern would be related to the incidence of GDM. The participants had a $75 \mathrm{~g}$ oral glucose tolerance test (OGTT) at first and second trimesters of pregnancy. Only participants with a normal OGTT at first trimester were included. The final number of participants of the current study was 251 ( $50 \%$ of all participants). This study was conducted according to the guidelines laid down in the Declaration of Helsinki and all procedures involving human subjects were approved by The Ethics Committee of the Department of Obstetrics and Gynecology of Helsinki and Uusimaa Hospital District. Written informed consent was obtained from all participants.

One or more pathological glucose values in a $75 \mathrm{~g} 2$-h OGTT (run by a central laboratory) led to GDM diagnosis. The diagnostic thresholds were: fasting plasma glucose concentration $\geq 5.3 \mathrm{mmol} / \mathrm{l}, \quad 1 \mathrm{~h}$ concentration $\geq 10.0 \mathrm{mmol} / 1$ and $2 \mathrm{~h}$ concentration $\geq 8.6 \mathrm{mmol} / \mathrm{l}^{(18)}$. The OGTT was conducted at 6-18 weeks of pregnancy and women with normal test results were included in the current study; the OGTT was then repeated at 24-28 weeks of pregnancy.

The HFII was used to measure the adherence of a diet to the NNR. The HFII has been thoroughly described previously ${ }^{(16)}$. We have shown that the HFII is valid for ranking participants according to their adherence to the NNR. The results of the validation study apply to pregnant obese women and women with previous GDM. The components of the HFII reflect the content of food-based guidelines of the $\mathrm{NNR}^{(15)}$. The HFII comprised eleven components covering the following food groups; vegetables, fruit and berries, high-fibre grains, fish, milk, cheese, cooking fat, fat spread, snacks, sugar-sweetened beverages and fast food (Table 1). The highest score was set to reflect the food-based guidelines of the NNR, whereas the lowest was indicative of a poor-quality diet. Whereas higher consumption of recommended foods resulted in higher scores, higher consumption of foods recommended in moderation (fast food, sugar-sweetened beverages and snacks) resulted in lower scores. Setting the maximum score was based on a priori assumption of the relative importance of the category for the overall diet quality. The HFII is a sum of the eleven components, and the scores range between 0 and 17. On the basis of the validation study ${ }^{(16)}$, the higher the HFII scores, the lower the intakes of fatty acids and SFA, and the higher the intakes of carbohydrate, dietary fibre, vitamin D, folate and MUFA and PUFA as percentages of total fat. In the validation study, the HFII was found to measure primarily three dimensions of the diet; Fats (ratio of low-fat:high-fat cheese, ratio of low-fat:high-fat milk, fatspread quality, cooking-fat quality), Healthy foods (fish, vegetables, fruit and berries, high-fibre grains) and Unhealthy foods (snacks, fast food, sugar-sweetened beverages). In the current study, HFII-sub-indices for each of these dimensions were created. A sum of the component scores of each dimension resulted in the sub-indices Fats, Healthy foods and Unhealthy foods (Table 1). The data for the HFII were derived from FFQ. The participants filled in the FFQ at the first visit to the study nurse at 6-18 weeks of pregnancy (mean gestational age 12.5 (SD 1.9) weeks) and at the second visit at 22-30 weeks of pregnancy (mean gestational age 26 (sD 1.9) weeks). 
Table 1. The Healthy Food Intake Index (HFII)-sub-indices among the components of the HFIl and their scoring studied among pregnant Finnish women at a high risk for gestational diabetes

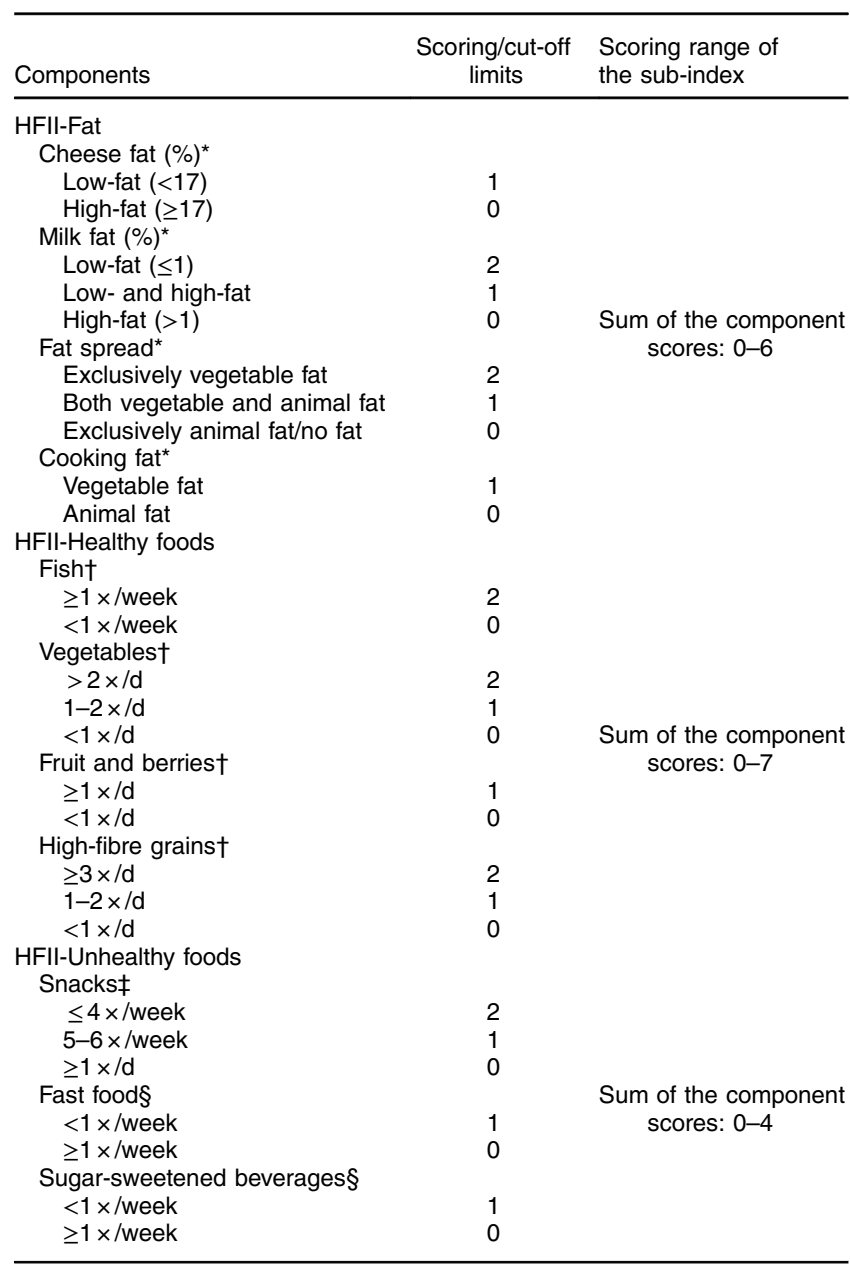

* Cut-off limits are based on the Nordic Nutrition Recommendations.

$\dagger$ Cut-off limits are based on the Finnish Nutrition Recommendations 2014 and a consensus-panel decision.

$\ddagger$ Cut-off limits are based on tertiles.

$\S$ Cut-off limits based on median.

The participants filled in a questionnaire on their health, lifestyle and history of pregnancies and pregnancy-related issues. Information on age, educational attainment, smoking (yes/no) and leisure time physical activity (LTPA) was also collected. LTPA was assessed by a self-report of weekly time spent on LTPA that makes a participant at least slightly out of breath and sweaty. BMI was calculated from the weight and height measured at the woman's first visit to the study nurse during pregnancy. Weight gain from the first to the second trimester was calculated from weights measured at first and second trimester visits.

\section{Statistical methods}

The descriptive analysis was performed in three groups according to the participants' baseline HFII, with the cut-off points set to $1 \mathrm{sD}$ from the mean HFII: category I (score range $0-7$ ), category II (score range 8-12) and category III (score range 13-17), respectively. Normality of the variables was tested by the Shapiro-Wilk $W$ test. The differences between women who did and did not develop GDM were analysed by Student's $t$ test, the Mann-Whitney $U$ test and Fisher's test. Difference in HFII at second trimester between women who developed GDM and women who did not develop GDM was tested by linear regression analysis. Association of change in the HFII and HFII-sub-indices with GDM was analysed by logistic regression analysis. The logistic regression analysis was adjusted for baseline HFII (or HFII-Fat/HFII-Healthy/HFII-Unhealthy), age, BMI, previous GDM (no/yes), parity (nulliparous/parous), weight change from the first to the second trimester, LTPA change and group assignment (control/intervention). Sensitivity analysis in groups of treatment (intervention/control), parity, previous GDM and low and high HFII was also performed by logistic regression analysis without adjustments. For the sensitivity analysis, HFII scores were divided into two groups using the median as a cut-off value. Stata 13.1 (StataCorp LP) statistical package was used for the analyses.

\section{Results}

At baseline (first trimester of pregnancy), the proportions of participants in the HFII categories (I, II and III) were 18 ( $n$ 46), 60 ( $n$ 151) and 22\% ( $n$ 54), respectively. In all, 18\% ( $n$ 46) of the participants developed GDM. From the lowest to the highest HFII category (I, II and III), the proportions of women who developed GDM were 15 ( $n$ 7), 17 ( $n$ 27) and 22\% ( $n$ 12), respectively. BMI was higher in women who did not develop GDM compared with women who developed GDM $(P=0 \cdot 006)$ (Table 2). Most women who developed GDM had a history of previous GDM (67.4\%) and this was different compared with women who did not develop GDM (34.1\%). No other statistically significant differences in baseline characteristics were observed between the women who developed GDM and women who did not.

The mean HFII score at baseline was 10.1 (95\% CI 9.7, 10.4), and the mean HFII change from the first to the second trimester of pregnancy improved by 0.35 (95\% CI 0.09, 0.62) points. The range of the HFII changes varied from -7 to 7 . The HFII score decreased, that is, the adherence to the NNR decreased, from the first to the second trimester of pregnancy in those who developed GDM (Fig. 1). On the contrary, the HFII improved in those who did not develop GDM. The odds for GDM were significantly lower with a higher change in the HFII score from the first to the second trimester of pregnancy (OR $0.83 ; 95 \%$ CI $0.69,0.99 ; P=0.043$ ) in the adjusted model (Fig. 2). From the other variables in the model, only previous GDM (OR 4.7; 95\% CI 1.28, 17.35; $P=0.02$ ) was significantly associated with GDM in the index pregnancy. When analysed in groups according to treatment (intervention/control), parity (nulliparous/parous), previous GDM (no/yes) or HFII at baseline (low/high), the association between HFII change and GDM were parallel between the groups (Fig. 3).

The fully adjusted odds for GDM were 0.72 (95\% CI 0.52 , 1.01) per one unit (point) increase in HFII-Fat from the first to the second trimester $(P=0.058)$ (Fig. 2). Changes in 
Table 2. Baseline (first trimester of pregnancy) demographic and clinical characteristics of pregnant women at a high risk for gestational diabetes (GDM) ( $n$ 251)

(Mean values and standard deviations; numbers and percentages)

\begin{tabular}{|c|c|c|c|c|c|}
\hline & \multicolumn{2}{|c|}{ GDM- (n 205) } & \multicolumn{2}{|c|}{$\mathrm{GDM}+(n$ 46) } & \multirow[b]{2}{*}{$P^{*}$} \\
\hline & Mean & SD & Mean & SD & \\
\hline Age (years) & $32 \cdot 1$ & 4.6 & $32 \cdot 9$ & 3.9 & 0.28 \\
\hline $\mathrm{BMl}$ at first trimester $\left(\mathrm{kg} / \mathrm{m}^{2}\right)$ & $32 \cdot 0$ & $5 \cdot 3$ & $29 \cdot 0$ & $6 \cdot 6$ & 0.006 \\
\hline Leisure time physical activity (min/week) & 144.1 & $218 \cdot 1$ & $105 \cdot 0$ & 141.5 & $0.46 \dagger$ \\
\hline Education (years) & 14.5 & 2.4 & $14 \cdot 3$ & 2.5 & 0.69 \\
\hline HFIl score (points) & $10 \cdot 0$ & $2 \cdot 8$ & 10.5 & $2 \cdot 8$ & 0.27 \\
\hline Fats (points) & 4.0 & 1.7 & $4 \cdot 3$ & 1.3 & 0.22 \\
\hline Healthy foods (points) & 3.7 & 1.7 & 3.6 & 1.6 & 0.82 \\
\hline Unhealthy foods (points) & $2 \cdot 3$ & 1.0 & $2 \cdot 6$ & 1.1 & 0.15 \\
\hline Fasting PG concentration ( $\mathrm{mmol} / \mathrm{l})$ & 4.88 & 0.24 & 4.88 & 0.25 & 0.90 \\
\hline $\mathrm{PG} 1 \mathrm{~h}$ postload $(\mathrm{mmol} / \mathrm{l})$ & $6 \cdot 80$ & 1.36 & 7.79 & 1.22 & $<0.001$ \\
\hline \multirow[t]{2}{*}{$\mathrm{PG} 2 \mathrm{~h}$ postload $(\mathrm{mmol} / \mathrm{l})$} & $5 \cdot 75$ & 1.07 & $6 \cdot 31$ & 1.23 & 0.002 \\
\hline & $n$ & $\%$ & $n$ & $\%$ & $P \ddagger$ \\
\hline Previous GDM & & & & & $<0.001$ \\
\hline No & 52 & 25.4 & 4 & 8.7 & \\
\hline Yes & 70 & 34.1 & 31 & 67.4 & \\
\hline Nulliparous & 83 & 40.5 & 11 & 23.9 & \\
\hline At least one parent has a history of diabetes (type 1 or 2 ) & 44 & 22 & 15 & $32 \cdot 6$ & 0.13 \\
\hline Mother has a history of GDM & 18 & 8.8 & 2 & $4 \cdot 3$ & $0.55 \S$ \\
\hline
\end{tabular}

GDM-, group of women who did not develop GDM; GDM+, group of women who developed GDM; HFII, Healthy Food Intake Index; PG, plasma glucose. * Tested by Student's $t$ test if not stated otherwise.

† Mann-Whitney $U$ test.

$\ddagger x^{2}$ test if not stated otherwise.

$\S$ Fisher's exact test.

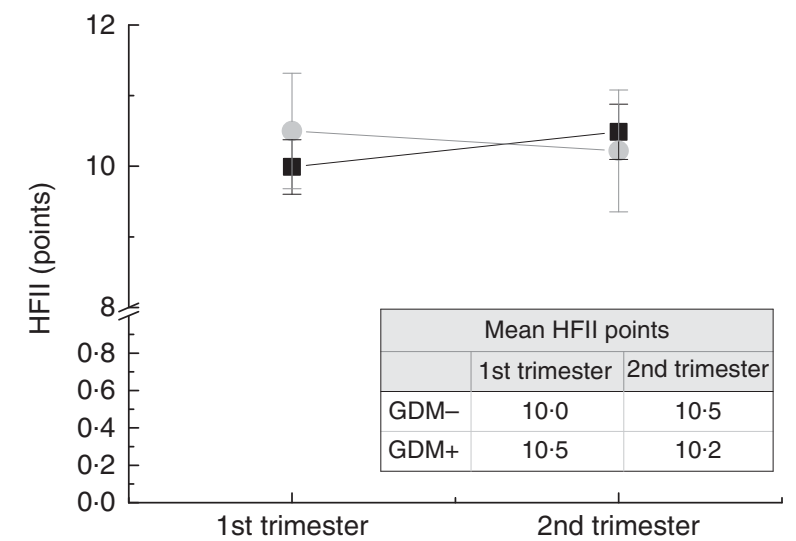

Fig. 1. Mean and $95 \% \mathrm{Cl}$ of the Healthy Food Intake Index (HFII) (range 0-17) at first and second trimesters of pregnancy in non-gestational diabetes (GDM) (GDM-, $-\square-)$ and GDM (GDM+, - - ) groups.

HFII-Healthy foods $(P=0.40)$ and in HFII-Unhealthy foods $(P=0.33)$ were not significantly associated with the risk for GDM.

\section{Discussion}

Our findings suggest that dietary changes towards the food guidelines of the NNR made during pregnancy may be related to a lower risk for GDM and that the HFII can be a practical tool for measuring the changes. The relationship between GDM and change in the HFII seem to be attributed mostly to a change in the HFII-fat score.

The intervention applied probably affected the dietary intake of the participants. However, finding the reasons behind the dietary changes were not our study aims; instead, it was the association between the changes and later occurrence of GDM. A possible confounder of the intervention could be changes in physical activity. We have addressed this by adjusting the analysis for change in LTPA. We moreover adjusted the analysis for intervention assignment (intervention/control group), thereby addressing a potential unknown effect of the intervention.

A source of bias could be the fact that those with a history of GDM have a markedly increased risk for GDM compared with women without a history of GDM. Thus, the effect of dietary changes could be different in women with and without a history of GDM. However, in a separate analysis according to GDM history, the results were similar in women with and without a history of GDM. The association between higher HFII change and a lower risk for GDM seemed to concern only parous and not multiparous women. This probably attributes, at least partly, to a smaller number of nulliparous compared with multiparous participants in the present study, and a higher prevalence of GDM among multiparous compared with nulliparous women. Thus, the data of multiparous women were probably more statistically powered compared with the data of nulliparous women.

Our approach provided novel information on the association between GDM and dietary changes made during pregnancy. According to observational studies, GDM is associated 


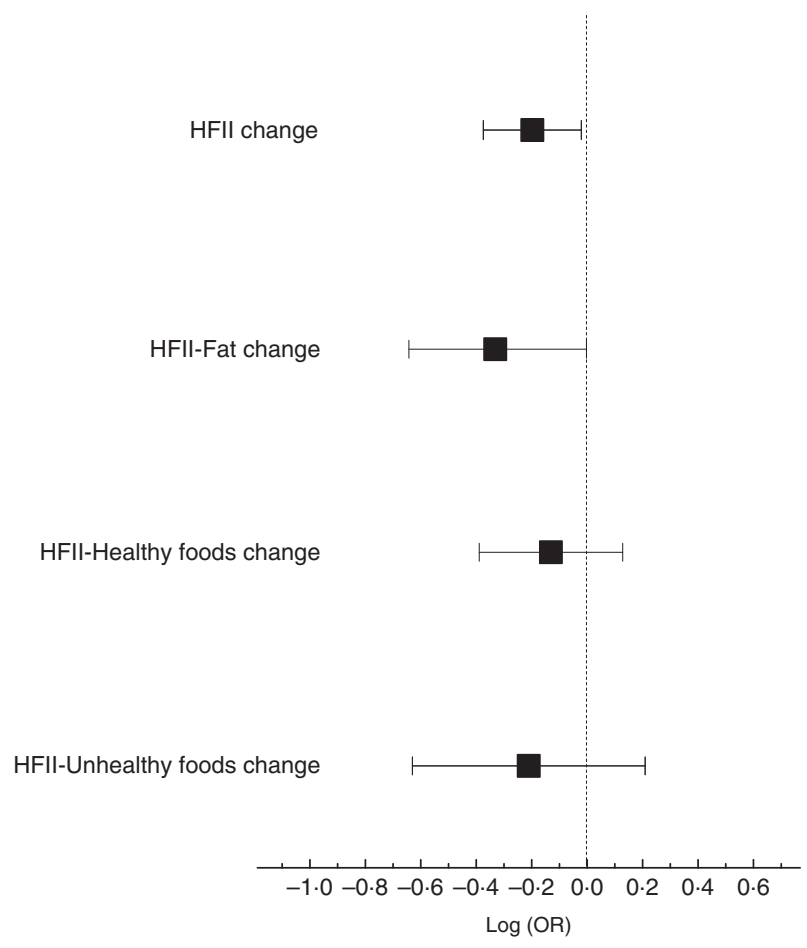

Fig. 2. Association of Healthy Food Intake Index (HFII) change (range from -7 to 7), HFII-Fat change (range from -4 to 5 ), HFII-Healthy foods change (range from -5 to 4 ) and HFII-Unhealthy foods change (range from -3 to 3 ) with the risk (log (OR) and $95 \% \mathrm{Cl}$ ) for gestational diabetes (GDM) in obese women and women with a history of GDM. The models were adjusted for baseline HFII (or HFII-Fat/HFII-Healthy foods/HFII-Unhealthy foods), age, BMI, previous GDM (no/yes), parity (nulliparous/parous), weight change from the first to the second trimester, leisure time physical activity change and group assignment (control/intervention).

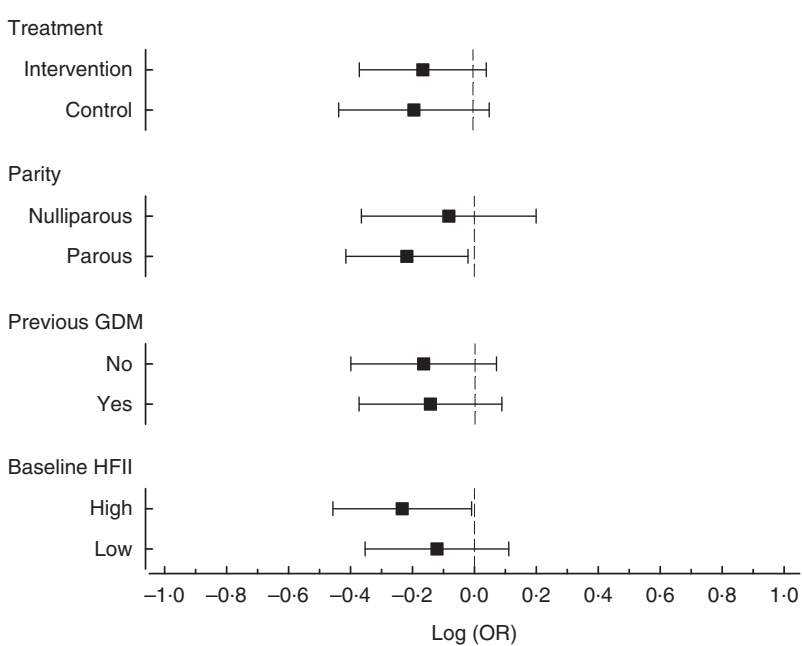

Fig. 3. Association of Healthy Food Intake Index (HFII) change (range from -7 to 7 ) with the risk (crude $\log (\mathrm{OR})$ and $95 \% \mathrm{Cl}$ ) for gestational diabetes (GDM) in obese women and women with a history of GDM in subgroups of the treatment group, parity, previous GDM and baseline HFII (median cut-off points).

with several acknowledged healthy dietary patterns with characteristics similar to those of a diet adherent to the $\mathrm{NNR}^{(6,10,19,20)}$. These studies have, however, either prospectively studied the association of GDM with a diet measured at one time point before or during pregnancy, or with a long-term dietary intake preceding pregnancy. Thus, a rationale for a comparison with the existing observational studies is limited.

Short-term effects of dietary change in preventing GDM have been studied mainly in diet or combined diet and exercise interventions ${ }^{(3,21,22)}$. Individual dietary intervention studies without additional intervention targets (such as physical exercise) have not succeeded in preventing GDM, possibly because of the small sample sizes. A meta-analysis found that dietary interventions lowered GDM incidence ${ }^{(23)}$, whereas another found no effect ${ }^{(24)}$. Although Rogozinska et al. ${ }^{(24)}$ did not find any effect of dietary counselling on GDM incidence, in a subanalysis they found that overweight and obese women benefitted from the intervention, whereas in studies including women of any weight the intervention was ineffective. The result supports our findings, because the majority of participants in our study were obese. Combined diet and physical activity intervention studies are more abundant than interventions concentrating exclusively on dietary counselling ${ }^{(3,21)}$. The majority of the several lifestyle intervention studies aimed at preventing GDM, however, have failed. Unfortunately, reports of these lifestyle interventions usually lack results on the effect of actual lifestyle changes on GDM risk, as was evident in a recent Cochrane systematic review ${ }^{(3)}$. Although seven out of the twelve included studies reported on dietary adherence to the intervention, none reported the level of adherence in relation to the intervention targets. Thus, whether a certain magnitude of lifestyle change would have been sufficient remained unclear. Two recent lifestyle interventions with diet and physical activity counselling have succeeded in preventing $\operatorname{GDM}^{(4,25)}$, which is in accordance with the current results. We recently reported that the RADIEL study was successful in preventing $\mathrm{GDM}^{(4)}$ but we did not yet report on the effects of the actual lifestyle changes on GDM. Changes in food intakes were seen in low-fat cheese and fish ${ }^{(26)}$. Subtle changes that were not detectable in other individual foods were taken into account in the HFII of the current study. The results of the RADIEL intervention and the present study suggest that compliance with dietary counselling on the basis of the NNR could result in a lower risk for GDM. The causes of the contradictory results of lifestyle intervention studies need further investigation. A somewhat surprising finding was the observation that women in the highest HFII category (who had the highest adherence to the NNR) had the highest GDM incidence. This probably reflects the inclusion criteria, which allowed participants who were non-obese but had a history of GDM. These women appeared to have the highest risk for $\mathrm{GDM}^{(4,27)}$. Because of the heterogeneity of the underlying mechanisms of $\mathrm{GDM}^{(27)}$, the same dietary strategy may not be equally effective among all high-risk women ${ }^{(28)}$. This area needs further studying.

The results suggest that for GDM prevention, the foods included in the HFII-Fat (milk fat $\%$, cheese fat $\%$, spread fat and cooking fat) may be the most important targets of dietary change during pregnancy. This may also reflect a possible higher accuracy of the HFII-Fat components compared with other components of the HFII. In the reproducibility analysis of our recent paper $^{(16)}$, the components of the HFII-fat showed slightly higher reproducibility compared with other components. 
The components measured choices between high- $v$. low-fat and vegetable $v$. animal fat products. HFII-Fat may measure a dietary aspect more constant over time than intake frequencies of foods, which the other components measured. Thus, the sub-indices may not be equally comparable with each other.

This study has some limitations. Originally the HFII was not designed to measure dietary change, and it may be rather insensitive for measuring change. This suggests that the magnitude of the relationship between the HFII and GDM could be even stronger than what was detected in the present study. In addition, all the components of the HFII do not measure change equally. For example, decreasing the frequency of snacks (candy, pastry, chocolate, chips and ice cream) from four times a week does not produce any change in the HFII score. Similarly, if one consumes vegetables once a day at baseline and increases it to two times, the HFII score remains unchanged. Subtle changes remaining unnoticed may result in differences in the ability of the HFII-sub-scores to detect changes. It is also important to notice that the HFII does not measure changes in all foods. Thus, some possibly relevant aspects of diets in relation to GDM may have remained unnoticed. The underlying FFQ for the HFII was not validated. However, the HFII was validated against $3-\mathrm{d}$ food records using the same $\mathrm{FFQ}^{(16)}$. As this is an observational study, we cannot conclude on causality because of possible residual confounding. In addition, LTPA was self-reported, which may not be a reliable measure of true physical activity ${ }^{(29)}$. The study group did not represent the general population but those at a high risk for GDM due to obesity and/or to a history of GDM. Nonetheless, these criteria concern a large group of the population; one-third of Finnish women at reproductive age are overweight or obese ${ }^{(30)}$. The results can, however, be generalised to only obese pregnant women and pregnant women with a history of GDM.

Strengths of the current study include a thoroughly validated HFII shown to measure adherence of diet to the NNR in a high GDM risk population. A longitudinal setting allowed us to study change within an individual instead of differences between individuals, which cannot be assumed to reflect true change. A prospective setting with dietary measures at two different time points provides stronger evidence of causality than a dietary measure at one single time point only. By testing and excluding those with pathological OGTT at baseline, we excluded the possible effect of the GDM diagnosis on diet and thus the possibility of reverse causality. The RADIEL main trial and the current study uniquely included participants who were non-obese but had a history of GDM. By including these women, we took into account the heterogeneity of the pathophysiology of $\mathrm{GDM}^{(27)}$.

\section{Conclusions}

Dietary change towards the NNR during pregnancy may be related to a lower risk of GDM. This supports the idea that GDM prevention by dietary changes started during early pregnancy could be effective. Well-powered interventions with detailed measures of intervention adherence, as well as descriptive studies on obstacles for intervention adherence are necessary. Studies on interaction of diet and different pathophysiological factors of GDM are also needed.

\section{Acknowledgements}

The authors are grateful to the women who participated as subjects, the study nurses, research dietitians and research scientists who contributed to this study.

This work was supported by the Ahokas Foundation, Finnish Foundation for Cardiovascular Disease, Special State Subsidy for Health Science Research of Helsinki University Central Hospital, Samfundet Folkhälsan, The Finnish Diabetes Research Foundation, Foundation for Medical Research Liv och Hälsa, State Provincial Office of Southern Finland and The Social Insurance Institution of Finland. The funders had no role in the design, analysis or writing of this article.

J. M., A. V., S. B. K, K. R., J. L., H. K., B. S.-L., J. G E. and M. E. designed and conducted the research. J. M., H. K. and M. E. performed statistical analysis. J. M., A. V. and M. E. wrote the paper. J. M. and M. E. had primary responsibility for final content. A. V., S. B. K., K. R., J. L., H. K., B. S.-L., J. G. E. and M. E. read and approved the final manuscript. S. B. K., K. R., J. L., H. K., B. S.-L. and J. G. E. critically revised the manuscript with expert advice and comments.

None of the authors has any conflicts of interest to declare.

\section{References}

1. Lamberg S, Raitanen J, Rissanen P, et al. (2012) Prevalence and regional differences of gestational diabetes mellitus and oral glucose tolerance tests in Finland. Eur J Public Health 22 , 278-280.

2. O'Sullivan EP, Avalos G, O'Reilly M, et al. (2011) Atlantic Diabetes in Pregnancy (DIP): the prevalence and outcomes of gestational diabetes mellitus using new diagnostic criteria. Diabetologia 54, 1670-1675.

3. Bain E, Crane M, Tieu J, et al. (2015) Diet and exercise interventions for preventing gestational diabetes mellitus. The Cochrane Database of Systematic Reviews 2015, issue 4, CD010443

4. Koivusalo SB, Rono K, Klemetti MM, et al. (2016) Gestational diabetes mellitus can be prevented by lifestyle intervention: The Finnish Gestational Diabetes Prevention Study (RADIEL): a randomized controlled trial. Diabetes Care 39, 24-30.

5. Jing X, Qiao R, Li M, et al. (2010) Gestational diabetes mellitus and the lifestyle and dietary structure of pregnant women: a case-control study. Wei Sheng Yan Jiu 39, 209-211.

6. Schoenaker DA, Soedamah-Muthu SS, Callaway LK, et al. (2015) Pre-pregnancy dietary patterns and risk of gestational diabetes mellitus: results from an Australian population-based prospective cohort study. Diabetologia 58, 2726-2735.

7. Tobias DK, Zhang C, Chavarro J, et al. (2012) Prepregnancy adherence to dietary patterns and lower risk of gestational diabetes mellitus. Am J Clin Nutr 96, 289-295.

8. Karamanos B, Thanopoulou A, Anastasiou E, et al. (2014) Relation of the Mediterranean diet with the incidence of gestational diabetes. Eur J Clin Nutr 68, 8-13.

9. Zhang C, Schulze M, Solomon C, et al. (2006) A prospective study of dietary patterns, meat intake and the risk of gestational diabetes mellitus. Diabetologia 49, 2604-2613.

10. He J, Yuan M, Chen NN, et al. (2015) Maternal dietary patterns and gestational diabetes mellitus: a large prospective cohort study in China. Br J Nutr 113, 1292-1300.

11. Schoenaker DAJM, Mishra GD, Callaway LK, et al. (2016) The role of energy, nutrients, foods, and dietary patterns in the development of gestational diabetes mellitus: a systematic review of observational studies. Diabetes Care 39, 16-23. 
12. Sacks FM, Obarzanek E, Windhauser MM, et al. (1995) Rationale and design of the Dietary Approaches to Stop Hypertension trial (DASH). A multicenter controlled-feeding study of dietary patterns to lower blood pressure. Ann Epidemiol 5, 108-118.

13. Hu FB (2002) Dietary pattern analysis: a new direction in nutritional epidemiology. Curr Opin Lipidol 13, 3-9.

14. Clutter Snyder D, Sloane R, Haines PS, et al. (2007) The Diet Quality Index-Revised: a tool to promote and evaluate dietary change among older cancer survivors enrolled in a home-based intervention trial. J Am Diet Assoc 107, 1519-1529.

15. Nordic Council of Ministers (2014) Nordic Nutrition Recommendations 2012 - Integrating Nutrition and Physical Activity, 5th ed. Copenhagen: Nordic Council of Ministers.

16. Meinila J, Valkama A, Koivusalo SB, et al. (2016) Healthy Food Intake Index (HFII) - validity and reproducibility in a gestational-diabetes-risk population. BMC Public Health 16, 680 .

17. Koivusalo SB, Rono K, Klemetti MM, et al. (2016) Gestational diabetes mellitus can be prevented by lifestyle intervention: The Finnish Gestational Diabetes Prevention Study (RADIEL): a randomized controlled trial. Diabetes Care 39, 24-30.

18. American Diabetes Association (2008) Standards of medical care in diabetes - 2008. Diabetes Care 31, Suppl. 1, S12-S54.

19. Shin D, Lee KW \& Song WO (2015) Dietary patterns during pregnancy are associated with risk of gestational diabetes mellitus. Nutrients 7, 9369-9382.

20. Tryggvadottir EA, Medek H, Birgisdottir BE, et al. (2016) Association between healthy maternal dietary pattern and risk for gestational diabetes mellitus. Eur J Clin Nutr 70, 237-242.

21. Tieu J, Crowther CA \& Middleton P (2008) Dietary advice in pregnancy for preventing gestational diabetes mellitus. The Cochrane Database of Systematic Reviews 2008, issue 16, CD006674.
22. Han S, Middleton P \& Crowther CA (2012) Exercise for pregnant women for preventing gestational diabetes mellitus. The Cochrane Database of Systematic Reviews 2012, issue 7 , CD009021.

23. Oostdam N, van Poppel MN, Wouters MG, et al. (2011) Interventions for preventing gestational diabetes mellitus: a systematic review and meta-analysis. J Womens Health (Larchmt) 20, 1551-1563.

24. Rogozinska E, Chamillard M, Hitman GA, et al. (2015) Nutritional manipulation for the primary prevention of gestational diabetes mellitus: a meta-analysis of randomised studies. PLOS ONE 10, e0115526.

25. Jing W, Huang Y, Liu X, et al. (2015) The effect of a personalized intervention on weight gain and physical activity among pregnant women in China. Int J Gynaecol Obstet 129, 138-141.

26. Valkama A, Koivusalo S, Lindstrom J, et al. (2016) The effect of dietary counselling on food intakes in pregnant women at risk for gestational diabetes: a secondary analysis of a randomised controlled trial RADIEL. Eur J Clin Nutr 70, 912-917.

27. Huvinen E, Grotenfelt NE, Eriksson JG, et al. (2016) Heterogeneity of maternal characteristics and impact on gestational diabetes (GDM) risk - implications for universal GDM screening? Ann Med 48, 52-58.

28. Grotenfelt NE, Wasenius NS, Rono K, et al. (2016) Interaction between rs10830963 polymorphism in MTNR1B and lifestyle intervention on occurrence of gestational diabetes. Diabetologia 59, 1655-1658.

29. Evenson KR, Chasan-Taber L, Symons Downs D, et al. (2012) Review of self-reported physical activity assessments for pregnancy: summary of the evidence for validity and reliability. Paediatr Perinat Epidemiol 26, 479-494.

30. National Institute for Health and Welfare (2013) Perinatal statistics: parturients deliveries and newborns 2012, no. 24. Helsinki: National Institute for Health and Welfare. 